\title{
Response Analysis of Frame Supporting Structure of Slope under Harmonic Vibration
}

\author{
Jian Duan, ${ }^{1,2}$ Zhi-xin Yan, ${ }^{1,2}$ Rui-jian Guo, ${ }^{1,2}$ and Zhi-hua Ren ${ }^{1,2,3}$ \\ ${ }^{1}$ School of Civil Engineering and Mechanics, Lanzhou University, Lanzhou 730000, China \\ ${ }^{2}$ Key Laboratory of Mechanics on Disaster and Environment in Western China (Lanzhou University), Ministry of Education, \\ Lanzhou 730000, China \\ ${ }^{3}$ Institute of Science and Technology of Yunnan Province Highway, Kunming 650051, China \\ Correspondence should be addressed to Zhi-xin Yan; yzx10@163.com
}

Received 16 June 2013; Revised 9 October 2013; Accepted 10 October 2013; Published 9 January 2014

Academic Editor: Evangelos J. Sapountzakis

Copyright (C) 2014 Jian Duan et al. This is an open access article distributed under the Creative Commons Attribution License, which permits unrestricted use, distribution, and reproduction in any medium, provided the original work is properly cited.

Based on certain assumptions, the dynamic mechanical model for frame supporting structure of slope is established, the dynamic equilibrium governing equation for vertical beam under forced vibration is derived, and hence its analytical solutions to harmonic forced vibration are obtained. What is more, the finite difference format and corresponding calculation procedure for vertical beam under forced vibration are given and programmed by using MATLAB language. In the case studies, comparative analyses have been performed to the response of vertical beam under horizontal harmonic forced vibration by using different calculating methods and with anchoring system damping effect neglected or considered. As a result, the feasibility, correctness, and characteristics of different methods can be revealed and the horizontal forced vibration law of vertical beam can be unveiled as well.

\section{Introduction}

Frame supporting techniques of slope have been widely applied due to their outstanding advantages [1]. The forcetransferring mechanism is that the frame supporting structure transmits the earth pressure or additional forces (such as seismic loading) it bears to the bolt, then transmits them to deep stable ground, and guarantees the safety of slope. It is obvious that frame supporting structure is a very important component in the whole system. Frame supporting structure always consists of horizontal beam and vertical beam, which are casted by concrete and shelved or mounted on slope surface. It functions by fixing the intersection of horizontal beam and vertical beam through anchor. Even though previous earthquake damage surveys have revealed that frame supporting techniques of slope show good seismic performance [2], the additional seismic stress triggered by strong earthquake is still high enough to cause the failure of frame supporting structure, such as inclined section shear failure or normal section bending failure, and cause secondary geological hazards. In terms of the mechanical characteristics of frame supporting structure of slope, quite a lot of researches have been conducted. For example, comparing with field tests, Yang et al. [3] have put up forward the calculating model and equation for frame supporting structure with anchor based on the principle of elastic foundation beam. Tian et al. [4] have given the finite difference format for the internal force calculation of frame supporting structure with bolt based on Winkler elastic foundation model and node deformation compatibility. Lin [5] has analyzed the influence of slope rate and anchoring force and so forth to the internal force through laboratory model test of frame supporting structure. Zhu et al. [6] have studied the distribution law of frame internal force and nodal force between horizontal beam and vertical beam by in situ test. Based on Winkler elastic foundation beam theory and certain assumptions, Dong et al. [7] have established a dynamic computing model for frame supporting structure with prestressed anchor and given the analytical solution to harmonic forced vibration by using modal analysis method. However, the mechanical characteristics of frame supporting structure of slope not only involve the mutual interaction among beams, ground, and anchors, but also should consider the deformation compatibility within the structure. What is 
more, current studies only focus on statistic problems, while few researches have paid attention to dynamic problems, and no related code can be referred to as well. Therefore, the dynamic behaviors of frame supporting structure of slope under harmonic forced vibration are explored in this paper, and this research can provide some theoretical support to the calculation of dynamic force and reinforcement of the structure.

\section{Dynamic Mechanical Model of Frame Supporting Structure of Slope}

2.1. Basic Assumptions. Under the horizontal seismic loading, the dynamic mechanical model of frame supporting structure of slope is established based on following assumptions.

(1) Beams and ground of slope are considered as continuous, isotropic, and elastic, and only the elastic dynamic problems are analyzed.

(2) Space torsional effect of beams is neglected; horizontal beam and vertical beam are viewed as independent continuous beam, respectively. Meanwhile, the mutual interaction among horizontal beam, vertical beam and anchor is considered through node deformation compatibility.

(3) Under the horizontal seismic loading $a_{g}^{\prime}(t)$ from slope bottom, the beams and the ground of slope keep contact all the time, and the interaction between them is simulated as horizontal linear spring model, the mechanical effect of which is similar to Winkler elastic foundation beam [8-13]. Therefore, the stiffness coefficient $k_{0}$ can be determined by referring to the theory of elastic foundation beam. The bottom of vertical beam is considered as sliding support which only subjected to vertical constraint, for its horizontal constraint is so slight that can be neglected.

(4) Influenced by slope height, the particle load acceleration of frame is $a_{g}(t)=\psi\left(y+H_{0}\right) a_{g}^{\prime}(t)$, in which the value for the amplification coefficient of acceleration $\psi\left(y+H_{0}\right)$ refers to literature [14], where $y$ is the vertical height from the particle to lower platform and $H_{0}$ is the vertical length from lower platform to slope bottom.

So the dynamic mechanical model of horizontal beam and vertical beam is almost the same, and the only difference between them lies in the characteristic of particle load acceleration function. More specifically, the corresponding parameter of horizontal beam $\psi\left(h_{i}+H_{0}\right)$ is constant. In other words, the horizontal seismic acceleration for the whole horizontal beam is the same at the same time. However, for the vertical beam, the corresponding parameter of slopeheight effect would change with the change of particle height; as a result the particle load acceleration along the vertical beam would vary at the same moment. Therefore, the dynamic response of horizontal beam can be viewed as a special case of vertical beam, namely, that the particle acceleration of beam is the same, so the analyses for vertical beam can be extended to horizontal beam. Dynamic response analysis for vertical beam is conducted as follows.

2.2. Dynamic Governing Equation. According to structural dynamic theory and above basic assumptions, the dynamic mechanical model of vertical beam is shown in Figure 1.

Based on the layout scheme of slope bolts, the vertical beam of the frame supporting structure can be divided into $n$ sections. To $i$ th section, the segment of infinitesimal length $d y / \sin \beta$ is chosen to perform the dynamic response analysis, just as the revelation of Figure 1. According to D'Alembert principle, the dynamic equilibrium equation for this segment of infinitesimal length can be expresses as

$$
Q_{i}+\frac{\partial Q_{i}}{\partial y} d y+\frac{\partial^{2} w_{i}}{\partial t^{2}} d m+\frac{k_{0} b w_{i} d y}{\sin \beta}=a_{g}(t) d m+Q_{i}
$$

where $Q_{i}$ and $w_{i}$ represent the shear force and horizontal displacement of vertical beam, respectively, $\beta$ is slope angle, and the mass of the segment of infinitesimal length is $d m=$ $(\rho b h / \sin \beta) d y$, where $b, h$, and $\rho$ are the width, height, and density of vertical beam, respectively. Equation (1) can be simplified as

$$
\frac{\partial Q_{i}}{\partial y}+\frac{\rho b h}{\sin \beta} \frac{\partial^{2} w_{i}}{\partial t^{2}}+\frac{k_{0} b}{\sin \beta} w_{i}=\frac{\rho b h}{\sin \beta} a_{g}(t) .
$$

Since $M_{i}=E I\left(\partial^{2} w_{i} / \partial y^{2}\right), Q_{i}=\partial M_{i} / \partial y=E I\left(\partial^{3} w_{i} / \partial y^{3}\right)$, and $I=b h^{3} / 12 \sin ^{3} \beta$, the dynamic equilibrium can be further simplified as

$$
\frac{\partial^{4} w_{i}}{\partial y^{4}}+\frac{\rho b h}{E I \sin \beta} \frac{\partial^{2} w_{i}}{\partial t^{2}}+\frac{k_{0} b}{E I \sin \beta} w_{i}=\frac{\rho b h}{E I \sin \beta} a_{g}(t) .
$$

Given that $\alpha_{1}=\rho b h / E I \sin \beta$, and $\alpha_{2}=k_{0} b / E I \sin \beta$, the dynamic governing equation can be transformed into

$$
\frac{\partial^{4} w_{i}}{\partial y^{4}}+\alpha_{1} \frac{\partial^{2} w_{i}}{\partial t^{2}}+\alpha_{2} w_{i}=\alpha_{1} a_{g}(t)
$$

2.3. Boundary Condition. According to the above basic assumptions, the boundary condition for the dynamic response of vertical beam could be obtained:

$$
\begin{gathered}
\left.M_{1}\right|_{y=0}=\left.E I \frac{\partial^{2} w_{1}}{\partial y^{2}}\right|_{y=0}=0, \\
\left.Q_{1}\right|_{y=0}=\left.E I \frac{\partial^{3} w_{1}}{\partial y^{3}}\right|_{y=0}=0, \\
\left.M_{n}\right|_{y=H}=\left.E I \frac{\partial^{2} w_{n}}{\partial y^{2}}\right|_{y=H}=0, \\
\left.Q_{n}\right|_{y=H}=\left.E I \frac{\partial^{3} w_{n}}{\partial y^{3}}\right|_{y=H}=0 .
\end{gathered}
$$

Meanwhile, according to the principle of deformation compatibility, in the outside endpoint of anchor, namely, that 


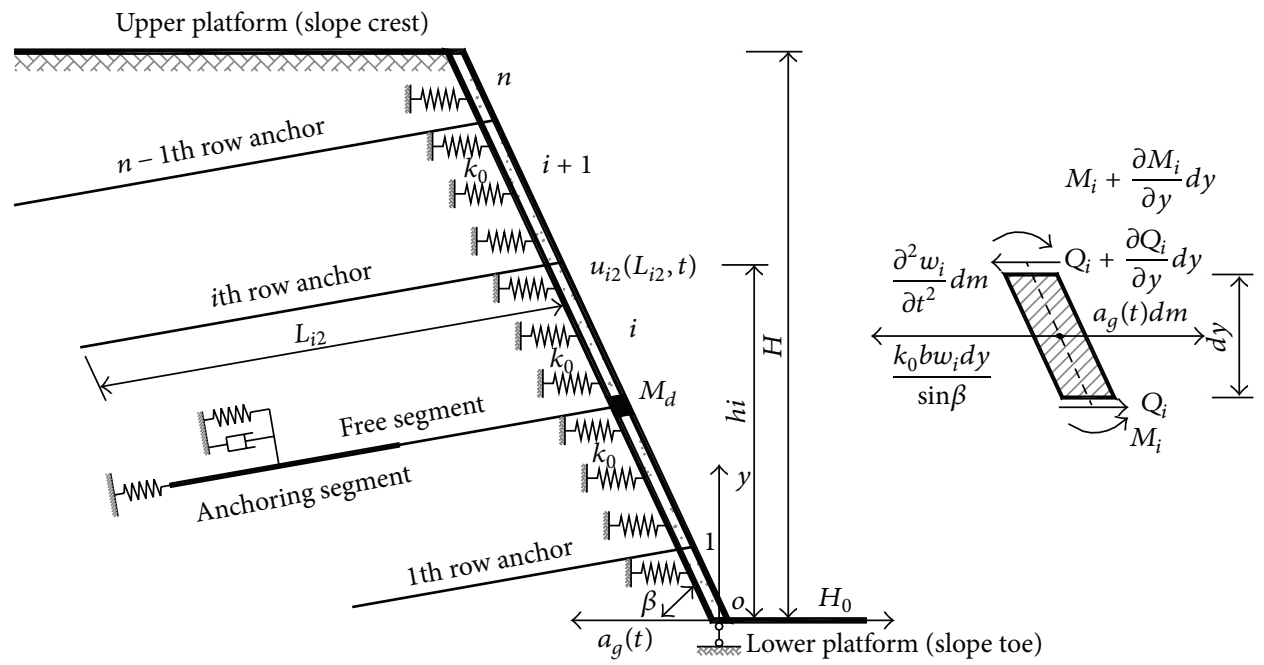

Figure 1: Dynamic mechanical model of vertical beam.

the demarcation point of vertical beam, the displacement $w_{i}$, rotation angle $\theta_{i}$, and bending moment $M_{i}$ should meet the following conditions:

$$
\begin{gathered}
\left.w_{i}\right|_{y=h_{i}}=\left.w_{i+1}\right|_{y=h_{i}}=u_{i 2}\left(L_{i 2}, t\right) \cos \varphi_{i} \quad(i=1 \sim n-1), \\
\left.\theta_{i}\right|_{y=h_{i}}=\left.\theta_{i+1}\right|_{y=h_{i}}=\left.\frac{\partial w_{i}}{\partial y}\right|_{y=h_{i}}=\left.\frac{\partial w_{i+1}}{\partial y}\right|_{y=h_{i}} \quad(i=1 \sim n-1), \\
\left.M_{i}\right|_{y=h_{i}}=\left.M_{i+1}\right|_{y=h_{i}} \\
=\left.E I \frac{\partial^{2} w_{i}}{\partial y^{2}}\right|_{y=h_{i}}=\left.E I \frac{\partial^{2} w_{i+1}}{\partial y^{2}}\right|_{y=h_{i}} \quad(i=1 \sim n-1),
\end{gathered}
$$

where $\varphi_{i}$ is the inclined angle of the $i$ th row anchor and $u_{i 2}\left(L_{i 2}, t\right)$ is the displacement of $i$ th row anchor of which, under horizontal seismic loading $a_{g}^{\prime}(t)$ from slope bottom, the specific formula can be found in the literature [15], which is based on some assumptions of using the Kelvin-Voigt model to simulate the interaction between anchoring section and the surrounding rock-soil mass, the inertial dynamic action of slope structure which exerts on the free section can be simplified as the equivalent lumped mass $M_{d}$ that acts on the end of free section, just as illustrated in the 2 th row anchor of Figure 1.

\section{Analytical Solutions to Vertical Beam under Harmonic Forced Vibration}

3.1. Theoretical Analysis. Coordination is considered and steady-state complex method is used to the derivation of harmonic forced vibration response of vertical beam. It is assumed that the solution to the governing equation (4) is $w_{i}^{\prime}(y, t)=W_{i}(y) e^{i \omega_{g} t}$. Then

$$
W_{i}^{\prime \prime \prime \prime}(y)+\left(\alpha_{2}-\alpha_{1} \omega_{g}^{2}\right) W_{i}(y)=\alpha_{1} a_{g}^{\prime} \psi\left(y+H_{0}\right) .
$$

Given that $\gamma_{1}=\beta(1-i), \gamma_{2}=\beta(1+i), \gamma_{3}=\beta(-1+i)$, and $\gamma_{4}=\beta(-1-i)$, the complex solution to $(7)$ is

$$
\begin{aligned}
W_{i}(y)= & C_{i 1} e^{\gamma_{1} y}+C_{i 2} e^{\gamma_{2} y}+C_{i 3} e^{\gamma_{3} y}+C_{i 4} e^{\gamma_{4} y} \\
& +\frac{\alpha_{1} a_{g}^{\prime} \psi\left(y+H_{0}\right)}{\alpha_{2}-\alpha_{1} \omega_{g}^{2}}
\end{aligned}
$$

where $C_{i 1}, C_{i 2}, C_{i 3}$, and $C_{i 4}$ are constant complex coefficients, which can be decided by specific boundary condition.

Likewise, the horizontal displacement, bending moment, and shear force of the $i$ th section in the vertical beam can be expressed as

$$
\begin{aligned}
& w_{i}^{\prime}(y, t) \\
& =W_{i}(y) e^{i \omega_{g} t} \\
& =\left(C_{i 1} e^{\gamma_{1} y}+C_{i 2} e^{\gamma_{2} y}+C_{i 3} e^{\gamma_{3} y}+C_{i 4} e^{\gamma_{4} y}+\frac{\alpha_{1} a_{g}^{\prime} \psi\left(y+H_{0}\right)}{\alpha_{2}-\alpha_{1} \omega_{g}^{2}}\right) \\
& \quad \times e^{i \omega_{g} t}, \\
& M_{i}^{\prime}(y, t) \\
& =E I\left(\gamma_{1}^{2} C_{i 1} e^{\gamma_{1} y}+\gamma_{2}^{2} C_{i 2} e^{\gamma_{2} y}+\gamma_{3}^{2} C_{i 3} e^{\gamma_{3} y}+\gamma_{4}^{2} C_{i 4} e^{\gamma_{4} y}\right) e^{i \omega_{g} t}, \\
& Q_{i}^{\prime}(y, t) \\
& =E I\left(\gamma_{1}^{3} C_{i 1} e^{\gamma_{1} y}+\gamma_{2}^{3} C_{i 2} e^{\gamma_{2} y}+\gamma_{3}^{3} C_{i 3} e^{\gamma_{3} y}+\gamma_{4}^{3} C_{i 4} e^{\gamma_{4} y}\right) e^{i \omega_{g} t} .
\end{aligned}
$$

Meanwhile, according to literature [15], the displacement response of the outside endpoint of anchor under horizontal harmonic loading can be simplified as $u_{i 2}^{\prime}\left(L_{i 2}, t\right)=\kappa_{i} e^{i \omega_{g} t}$. 
It is assumed that $U_{i}\left(h_{i}\right)=\kappa_{i} \cos \varphi_{i}$. Substitute them into boundary condition equations (5) and (6). Then

$$
\begin{gathered}
\gamma_{1}^{2} C_{11}+\gamma_{2}^{2} C_{12}+\gamma_{3}^{2} C_{13}+\gamma_{4}^{2} C_{14}=0, \\
\gamma_{1}^{3} C_{11}+\gamma_{2}^{3} C_{12}+\gamma_{3}^{3} C_{13}+\gamma_{4}^{3} C_{14}=0, \\
C_{i 1} e^{\gamma_{1} h_{i}}+C_{i 2} e^{\gamma_{2} h_{i}}+C_{i 3} e^{\gamma_{3} h_{i}}+C_{i 4} e^{\gamma_{4} h_{i}} \\
+\frac{\alpha_{1} a_{g}^{\prime} \psi\left(y+H_{0}\right)}{\alpha_{2}-\alpha_{1} \omega_{g}^{2}}=U_{i}\left(h_{i}\right), \\
C_{(i+1) 1} e^{\gamma_{1} h_{i}}+C_{(i+1) 2} e^{\gamma_{2} h_{i}}+C_{(i+1) 3} e^{\gamma_{3} h_{i}} \\
+C_{(i+1) 4} e^{\gamma_{4} h_{i}}+\frac{\alpha_{1} a_{g}^{\prime} \psi\left(y+H_{0}\right)}{\alpha_{2}-\alpha_{1} \omega_{g}^{2}}=U_{i}\left(h_{i}\right), \\
\gamma_{1} C_{i 1} e^{\gamma_{1} h_{i}}+\gamma_{2} C_{i 2} e^{\gamma_{2} h_{i}}+\gamma_{3} C_{i 3} e^{\gamma_{3} h_{i}}+\gamma_{4} C_{i 4} e^{\gamma_{4} h_{i}} \\
=\gamma_{1} C_{(i+1) 1} e^{\gamma_{1} h_{i}}+\gamma_{2} C_{(i+1) 2} e^{\gamma_{2} h_{i}}+\gamma_{3} C_{(i+1) 3} e^{\gamma_{3} h_{i}} \\
+\gamma_{4} C_{(i+1) 4} e^{\gamma_{4} h_{i}},
\end{gathered}
$$

$$
\begin{gathered}
\gamma_{1}^{2} C_{i 1} e^{\gamma_{1} h_{i}}+\gamma_{2}^{2} C_{i 2} e^{\gamma_{2} h_{i}}+\gamma_{3}^{2} C_{i 3} e^{\gamma_{3} h_{i}}+\gamma_{4}^{2} C_{i 4} e^{\gamma_{4} h_{i}} \\
=\gamma_{1}^{2} C_{(i+1) 1} e^{\gamma_{1} h_{i}}+\gamma_{2}^{2} C_{(i+1) 2} e^{\gamma_{2} h_{i}}+\gamma_{3}^{2} C_{(i+1) 3} e^{\gamma_{3} h_{i}} \\
+\gamma_{4}^{2} C_{(i+1) 4} e^{\gamma_{4} h_{i}}, \\
\gamma_{1}^{2} C_{n 1} e^{\gamma_{1} H}+\gamma_{2}^{2} C_{n 2} e^{\gamma_{2} H}+\gamma_{3}^{2} C_{n 3} e^{\gamma_{3} H}+\gamma_{4}^{2} C_{n 4} e^{\gamma_{4} H}=0, \\
\gamma_{1}^{3} C_{n 1} e^{\gamma_{1} H}+\gamma_{2}^{3} C_{n 2} e^{\gamma_{2} H}+\gamma_{3}^{3} C_{n 3} e^{\gamma_{3} H}+\gamma_{4}^{3} C_{n 4} e^{\gamma_{4} H}=0 .
\end{gathered}
$$

The above linear equations can be solved by matrix method. It is assumed that $A_{i}=e^{\gamma_{1} h_{i}}, B_{i}=e^{\gamma_{2} h_{i}}, C_{i}=e^{\gamma_{3} h_{i}}$, $D_{i}=e^{\gamma_{4} h_{i}}$, and $E_{i}=U_{i}\left(h_{i}\right)-\left(\alpha_{1} a_{g}^{\prime} \psi\left(y+H_{0}\right) /\left(\alpha_{2}-\alpha_{1} \omega_{g}^{2}\right)\right)$. Then (10) can be expressed as matrix format:

$$
C_{4 n \times 1}=B_{4 n \times 4 n}^{-1} A_{4 n \times 1},
$$

where

$$
\begin{aligned}
& A=\left[\begin{array}{llllllllllllllllll}
0 & 0 & E_{1} & E_{1} & 0 & 0 & \cdots & E_{i} & E_{i} & 0 & 0 & \cdots & E_{n-1} & E_{n-1} & 0 & 0 & 0 & 0
\end{array}\right]^{T}, \\
& C=\left[\begin{array}{llllllllllllll}
C_{11} & C_{12} & C_{13} & C_{14} & \cdots & C_{i 1} & C_{i 2} & C_{i 3} & C_{i 4} & \cdots & C_{n 1} & C_{n 2} & C_{n 3} & C_{n 4}
\end{array}\right]^{T},
\end{aligned}
$$

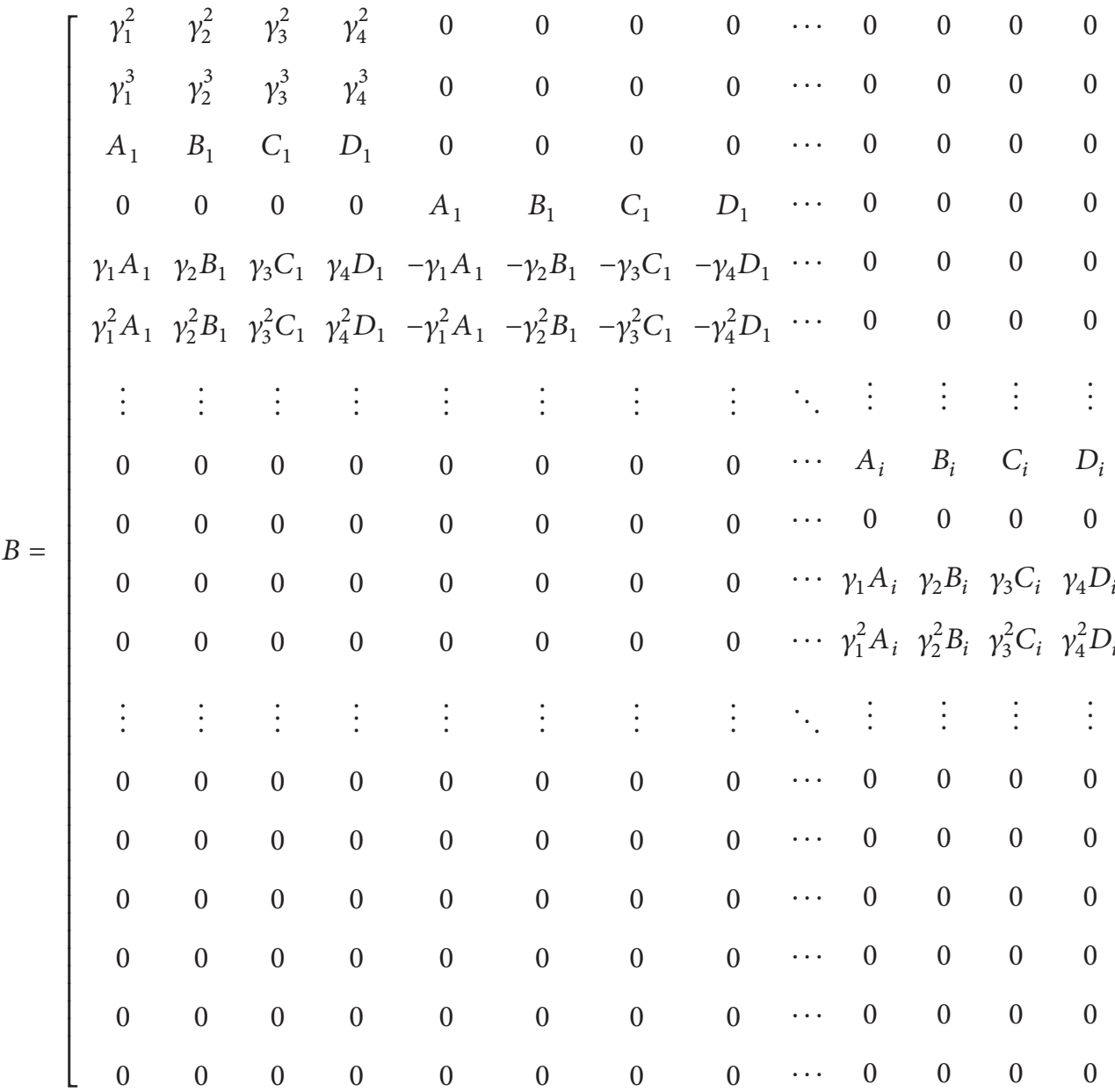




$\left.\begin{array}{ccccccccccccc}0 & 0 & 0 & 0 & \cdots & 0 & 0 & 0 & 0 & 0 & 0 & 0 & 0 \\ 0 & 0 & 0 & 0 & \cdots & 0 & 0 & 0 & 0 & 0 & 0 & 0 & 0 \\ 0 & 0 & 0 & 0 & \cdots & 0 & 0 & 0 & 0 & 0 & 0 & 0 & 0 \\ 0 & 0 & 0 & 0 & \cdots & 0 & 0 & 0 & 0 & 0 & 0 & 0 & 0 \\ 0 & 0 & 0 & 0 & \cdots & 0 & 0 & 0 & 0 & 0 & 0 & 0 & 0 \\ 0 & 0 & 0 & 0 & \cdots & 0 & 0 & 0 & 0 & 0 & 0 & 0 & 0 \\ \vdots & \vdots & \vdots & \vdots & \ddots & \vdots & \vdots & \vdots & \vdots & \vdots & \vdots & \vdots & \vdots \\ 0 & 0 & 0 & 0 & \cdots & 0 & 0 & 0 & 0 & 0 & 0 & 0 & 0 \\ A_{i} & B_{i} & C_{i} & D_{i} & \cdots & 0 & 0 & 0 & 0 & 0 & 0 & 0 & 0 \\ -\gamma_{1} A_{i} & -\gamma_{2} B_{i} & -\gamma_{3} C_{i} & -\gamma_{4} D_{i} & \cdots & 0 & 0 & 0 & 0 & 0 & 0 & 0 & 0 \\ -\gamma_{1}^{2} A_{i}-\gamma_{2}^{2} B_{i} & -\gamma_{3}^{2} C_{i} & -\gamma_{4}^{2} D_{i} & \cdots & 0 & 0 & 0 & 0 & 0 & 0 & 0 & 0 \\ \vdots & \vdots & \vdots & \vdots & \ddots & \vdots & \vdots & \vdots & \vdots & \vdots & \vdots & \vdots & \vdots \\ 0 & 0 & 0 & 0 & \cdots & A_{n-1} & B_{n-1} & C_{n-1} & D_{n-1} & 0 & 0 & 0 & 0 \\ 0 & 0 & 0 & 0 & \cdots & 0 & 0 & 0 & 0 & A_{n-1} & B_{n-1} & C_{n-1} & D_{n-1} \\ 0 & 0 & 0 & 0 & \cdots & \gamma_{1} A_{n-1} & \gamma_{2} B_{n-1} & \gamma_{3} C_{n-1} & \gamma_{4} D_{n-1} & -\gamma_{1} A_{n-1} & -\gamma_{2} B_{n-1} & -\gamma_{3} C_{n-1} & -\gamma_{4} D_{n-1} \\ 0 & 0 & 0 & 0 & \cdots & \gamma_{1}^{2} A_{n-1} & \gamma_{2}^{2} B_{n-1} & \gamma_{3}^{2} C_{n-1} & \gamma_{4}^{2} D_{n-1} & -\gamma_{1}^{2} A_{n-1} & -\gamma_{2}^{2} B_{n-1} & -\gamma_{3}^{2} C_{n-1} & -\gamma_{4}^{2} D_{n-1} \\ 0 & 0 & 0 & 0 & \cdots & 0 & 0 & 0 & 0 & \gamma_{1}^{2} A_{n} & \gamma_{2}^{2} B_{n} & \gamma_{3}^{2} C_{n} & \gamma_{4}^{2} D_{n} \\ 0 & 0 & 0 & 0 & \cdots & 0 & 0 & 0 & 0 & \gamma_{1}^{3} A_{n} & \gamma_{2}^{3} B_{n} & \gamma_{3}^{3} C_{n} & \gamma_{4}^{3} D_{n}\end{array}\right]$.

After getting constant complex coefficient $C_{4 n \times 1}$, it is assumed that $C_{i 1}=c_{i 1}+c_{i 2} i, C_{i 2}=c_{i 3}+c_{i 4} i, C_{i 3}=c_{i 5}+c_{i 6} i$, and $C_{i 4}=c_{i 7}+c_{i 8} i$. Substituting them into (9), the displacement can be simplified as

$$
\begin{aligned}
& w_{i}^{\prime}(y, t) \\
& =\left(c_{i 1} e^{y \beta}+c_{i 2} e^{y \beta} i\right) \\
& \quad \times\left[\cos \left(\omega_{g} t-y \beta\right)+i \sin \left(\omega_{g} t-y \beta\right)\right] \\
& +\left(c_{i 3} e^{y \beta}+c_{i 4} e^{y \beta} i\right)\left[\cos \left(\omega_{g} t+y \beta\right)+i \sin \left(\omega_{g} t+y \beta\right)\right] \\
& +\left(c_{i 5} e^{-y \beta}+c_{i 6} e^{-y \beta} i\right)\left[\cos \left(\omega_{g} t+y \beta\right)+i \sin \left(\omega_{g} t+y \beta\right)\right] \\
& +\left(c_{i 7} e^{-y \beta}+c_{i 8} e^{-y \beta} i\right)\left[\cos \left(\omega_{g} t-y \beta\right)+i \sin \left(\omega_{g} t-y \beta\right)\right] \\
& +\frac{\alpha_{1} a_{g}^{\prime} \psi\left(y+H_{0}\right)}{\alpha_{2}-\alpha_{1} \omega_{g}^{2}}\left(\cos \omega_{g} t+i \sin \omega_{g} t\right) .
\end{aligned}
$$

According to the calculating principle of complex method, the corresponding imaginary part is the steady-state solution to the mechanical response of vertical beam under harmonic seismic loading. Then

$$
\begin{aligned}
& w_{i}(y, t) \\
& =e^{\beta y}\left[c_{i 1} \sin \left(\omega_{g} t-\beta y\right)+c_{i 2} \cos \left(\omega_{g} t-\beta y\right)\right. \\
& \left.\quad+c_{i 3} \sin \left(\omega_{g} t+\beta y\right)+c_{i 4} \cos \left(\omega_{g} t+\beta y\right)\right] \\
& +e^{-\beta y}\left[c_{i 5} \sin \left(\omega_{g} t+\beta y\right)+c_{i 6} \cos \left(\omega_{g} t+\beta y\right)\right.
\end{aligned}
$$

$$
\begin{aligned}
& \left.+c_{i 7} \sin \left(\omega_{g} t-\beta y\right)+c_{i 8} \cos \left(\omega_{g} t-\beta y\right)\right] \\
& +\frac{\alpha_{1} a_{g}^{\prime} \psi\left(y+H_{0}\right)}{\alpha_{2}-\alpha_{1} \omega_{g}^{2}} \sin \omega_{g} t
\end{aligned}
$$$$
M_{i}(y, t)
$$$$
=2 \beta^{2} E I\left\{e ^ { \beta y } \left[-c_{i 1} \cos \left(\omega_{g} t-\beta y\right)+c_{i 2} \sin \left(\omega_{g} t-\beta y\right)\right.\right.
$$$$
\left.+c_{i 3} \cos \left(\omega_{g} t+\beta y\right)-c_{i 4} \sin \left(\omega_{g} t+\beta y\right)\right]
$$$$
+e^{-\beta y}\left[-c_{i 5} \cos \left(\omega_{g} t+\beta y\right)+c_{i 6} \sin \left(\omega_{g} t+\beta y\right)\right.
$$$$
\left.\left.+c_{i 7} \cos \left(\omega_{g} t-\beta y\right)-c_{i 8} \sin \left(\omega_{g} t-\beta y\right)\right]\right\},
$$$$
Q_{i}(y, t)
$$$$
=2 \beta^{3} E I\left\{e ^ { \beta y } \left[\left(c_{i 2}-c_{i 1}\right) \sin \left(\omega_{g} t-\beta y\right)\right.\right.
$$$$
-\left(c_{i 1}+c_{i 2}\right) \cos \left(\omega_{g} t-\beta y\right)
$$$$
-\left(c_{i 3}+c_{i 4}\right) \sin \left(\omega_{g} t+\beta y\right)
$$$$
\left.+\left(c_{i 3}-c_{i 4}\right) \cos \left(\omega_{g} t+\beta y\right)\right]
$$$$
+e^{-\beta y}\left[\left(c_{i 5}-c_{i 6}\right) \sin \left(\omega_{g} t+\beta y\right)\right.
$$$$
+\left(c_{i 5}+c_{i 6}\right) \cos \left(\omega_{g} t+\beta y\right)
$$$$
+\left(c_{i 7}+c_{i 8}\right) \sin \left(\omega_{g} t-\beta y\right)
$$$$
\left.\left.+\left(c_{i 8}-c_{i 7}\right) \cos \left(\omega_{g} t-\beta y\right)\right]\right\} \text {. }
$$ 
3.2. Case Studies. Take a slope as an example. Its height is $12 \mathrm{~m}$, the slope angle is $65^{\circ}$, and its physical and mechanical parameters are $\rho_{s}=1600 \mathrm{~kg} \cdot \mathrm{m}^{-3}, E_{s}=0.12 \mathrm{MPa}, \mu_{s}=$ $0.3, c_{s}=20 \mathrm{kPa}$, and $\phi_{s}=25^{\circ}$. The seismic precautionary intensity in the local region is 8 degrees and the peak ground acceleration is $0.2 \mathrm{~g}$. The dynamic safety factor is 1.015 calculated by Fellenius method; hence its support is needed. A preliminary supporting scheme is using fixed end anchors and frame supporting structure. The dynamic safety factor is considered as 1.2, calculated by the pseudostatic method, and the design scheme is shown in Figure 2. The anchor uses category II steel bar; diameter size is $28 \mathrm{~mm}$, and its space is $3.3 \mathrm{~m}$; the $\operatorname{dip} \varphi=10^{\circ}$; four rows of anchors have been arranged; both lengths of anchoring section and free section are $5 \mathrm{~m}$. The mortar grade M30 is used as bonding agent; the diameter of anchor hole is $D=130 \mathrm{~mm}$. The section size of the frame beam is $b \times h=0.45 \times 0.5 \mathrm{~m}$; concrete is grade C 30 , $\rho=2500 \mathrm{~kg} / \mathrm{m}^{3} ; E=25.5 \mathrm{GPa}$. Earthquake acceleration is $a_{g}^{\prime}(t)=a_{g}^{\prime} \sin \omega_{g} t=2 \sin 4 \pi t$, the duration is $2.5 \mathrm{~s}$, and $k_{0}=2 \times 10^{3} \mathrm{kN} / \mathrm{m}^{3}$.

From Figures 3, 4, and 5, the displacement, bending moment, and shear force response of vertical beam have been given when damping effect of anchorage system is neglected, while from Figures 6, 7, 8, 9, 10, 11, 12, and 13, corresponding response has been given at special sections (intersection and midspan section) and typical moment (according to moment of acceleration peak). It is found that the displacement, bending moment, and shear force response of vertical beam vibrate synchronously with earthquake. The law of bending moment and shear force response shows agreement with the harmonic forced vibration characteristic of two-end cantilevered continuous beam, and the anchor to the frame supporting structure is just like the bearing to the continuous beam. More specifically, the bending moment response at intersection opposite to the one at midspan section and the shear force response show a jumping from the left hand to the right hand at the intersection of vertical beam, just as illustrated in Figures 12 and 13. What is more, the cantilevered length at the two ends of vertical beam has noticeable influence on the mechanical response of frame supporting structure. The larger the cantilevered length is, the more the bending moment of intersection is, and the greater the difference of bending moment between intersection and midspan section is. Examining the frame supporting structure independently, the bending moment can keep good balance for the whole vertical beam when its cantilevered length is 0.2 or 0.25 times the anchor adjacent spacing, referring to the design experience of two-end cantilevered continuous beam. However, the cantilevered length of vertical beam is still related to the arranging scheme of anchors; actually the length even decides the arrangement of anchors and directly influences the slope supporting effect of anchors. Hence, in specific engineering design, the determination of cantilevered length should also consider the factors such as mechanical characteristic of vertical beam and slope supporting effect of anchors.

Just as shown from Figures 14, 15, and 16, the displacement, bending moment, and shear force response of vertical beam with damping effect considered have been given as well. The overall trend is similar to the previous one with damping effect neglected. When considering anchorage system damping effect, the solutions can be obtained by using steady-state complex method, and the peak values of the displacement, bending moment, and shear force are smaller than the ones with damping effect neglected. Even though the response shows some attenuation characteristic of amplitude in terms of magnitude, it does not demonstrate the attenuation characteristic of amplitude in terms of time. What is more, the mechanical responses show certain initial phases, which can be attributed to the dynamic behaviors of vertical beam under steady-state vibration with damping.

\section{Finite Difference Method for Vertical Beam under Forced Vibration}

4.1. Finite Difference Format for Dynamic Response Analysis. The numerical solutions to dynamic governing equation (4) could be also achieved by using finite difference method. According to the basic principles of finite difference method [16], the research area $A(0 \leq t \leq T, 0 \leq y \leq H)$ can be divided into $n$ subregions $A_{i}$. The whole region of $A_{i}$ can be redivided into many grids $A_{i \Delta t \Delta l_{1}}\left(M, N_{i}-N_{i-1}+1\right)$, which is shown in Figure 17. Meanwhile, in order to guarantee the convergence and stability of calculation, the ratio of the grids should meet the following condition: $r_{i}=\Delta t / \Delta^{2} l_{i} \leq \sqrt{\alpha_{1}}(i=1 \sim n)$.

The governing equation (4) can be expanded according to central difference formula, and the difference format of dynamic equation for grid node $w_{i}(j, k)$ can be obtained:

$$
\begin{aligned}
& \left(6 w_{i}(j, k)-4\left[w_{i}(j, k+1)+w_{i}(j, k-1)\right]\right. \\
& \left.+w_{i}(j, k+2)+w_{i}(j, k-2)\right)\left(\Delta l_{i}^{4}\right)^{-1} \\
& +\alpha_{1} \frac{w_{i}(j+1, k)+w_{i}(j-1, k)-2 w_{i}(j, k)}{\Delta t^{2}} \\
& +\alpha_{2} w_{i}(j, k)=\alpha_{1} \psi_{i}(k) a_{g}^{\prime}[j \Delta t],
\end{aligned}
$$

where $\psi_{i}(k)=\psi\left[\left(k-N_{i-1}\right) \Delta l_{i}+\sum_{z=1}^{z=i-1} \Delta l_{z}\left(N_{z}-N_{z-1}\right)+H_{0}\right]$.

Likewise, the boundary equations (5) and (6) can be expanded by endpoint derivative differential formula, and the corresponding differential equation can be attained:

$$
\begin{aligned}
& E I \frac{2 w_{1}(j, 0)-5 w_{1}(j, 1)+4 w_{1}(j, 2)-w_{1}(j, 3)}{\Delta l_{1}^{2}}=0, \\
& E I \frac{-w_{1}(j, 0)+3 w_{1}(j, 1)-3 w_{1}(j, 2)+w_{1}(j, 3)}{\Delta l_{1}^{3}}=0, \\
& \quad E I\left(2 w_{n}\left(j, N_{n}\right)-5 w_{n}\left(j, N_{n}-1\right)\right. \\
& \left.\quad+4 w_{n}\left(j, N_{n}-2\right)-w_{n}\left(j, N_{n}-3\right)\right)\left(\Delta l_{n}^{2}\right)^{-1}=0 \\
& E I\left(w_{n}\left(j, N_{n}\right)-3 w_{n}\left(j, N_{n}-1\right)\right. \\
& \left.\quad+3 w_{n}\left(j, N_{n}-2\right)-w_{n}\left(j, N_{n}-3\right)\right)\left(\Delta l_{n}^{3}\right)^{-1}=0 \\
& w_{i}\left(j, N_{i}\right)=w_{i+1}\left(j, N_{i}\right)=u_{i 2}\left(L_{i 2}, j \Delta t\right) \cos \varphi_{i},
\end{aligned}
$$




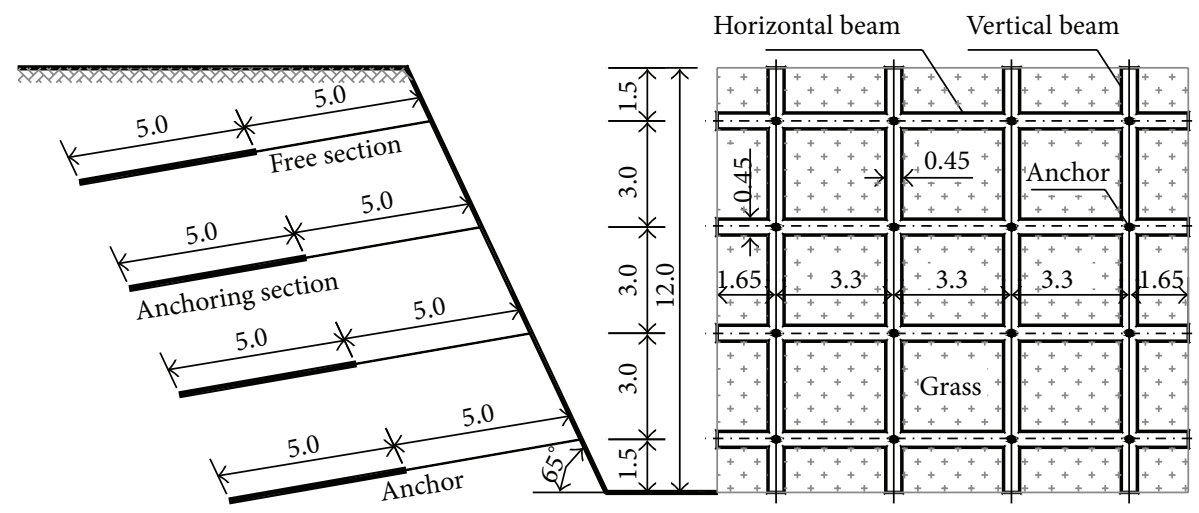

FIGURE 2: Design scheme for slope (unit: $\mathrm{m}$ ).

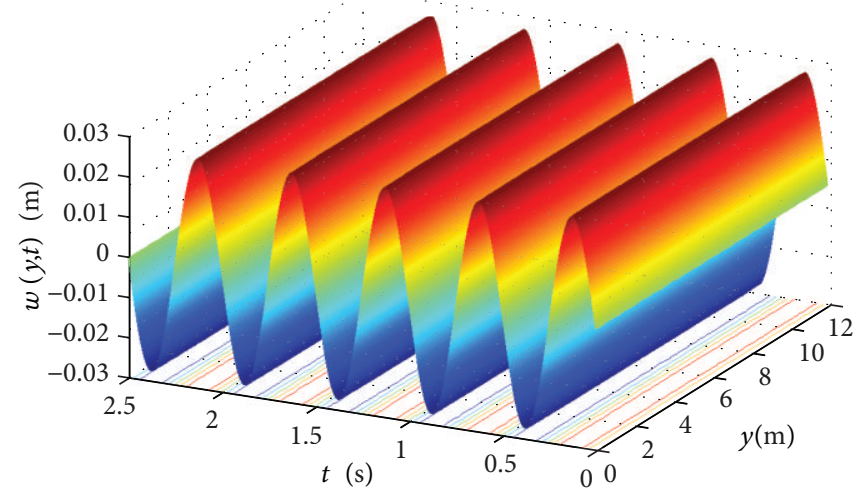

FIGURE 3: Displacement response of vertical beam.

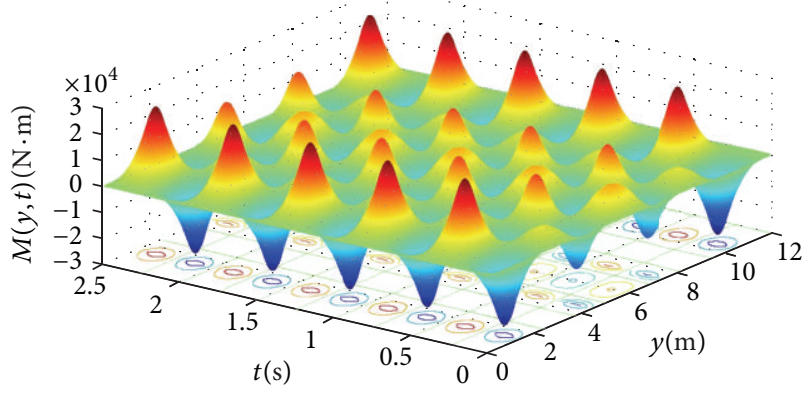

Figure 4: Bending moment response of vertical beam.

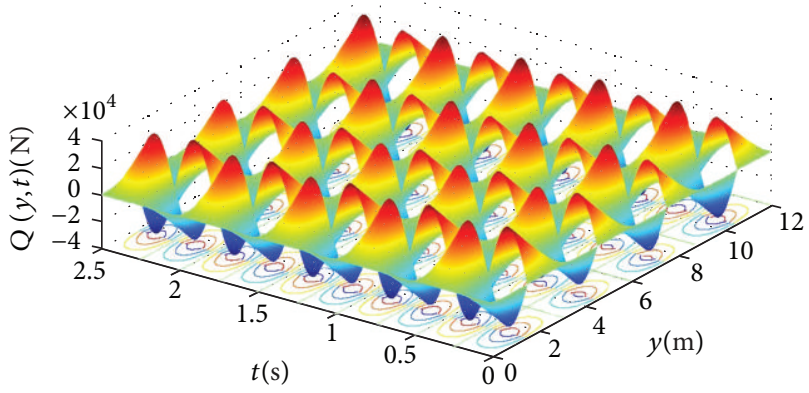

FIGURE 5: Shear force response of vertical beam.

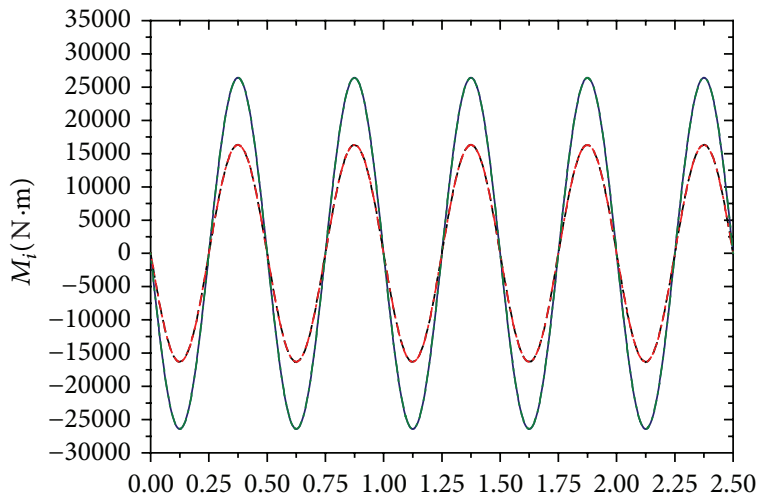

$t(\mathrm{~s})$

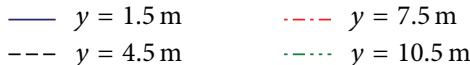

FIGURE 6: Bending moment response at intersection of vertical beam.

$$
\begin{aligned}
& \frac{2 w_{i}\left(j, N_{i}\right)-3 w_{i}\left(j, N_{i}-1\right)+w_{i}\left(j, N_{i}-2\right)}{\Delta l_{i}} \\
& =\frac{-2 w_{i+1}\left(j, N_{i}\right)+3 w_{i+1}\left(j, N_{i}+1\right)-w_{i+1}\left(j, N_{i}+2\right)}{\Delta l_{i+1}},
\end{aligned}
$$




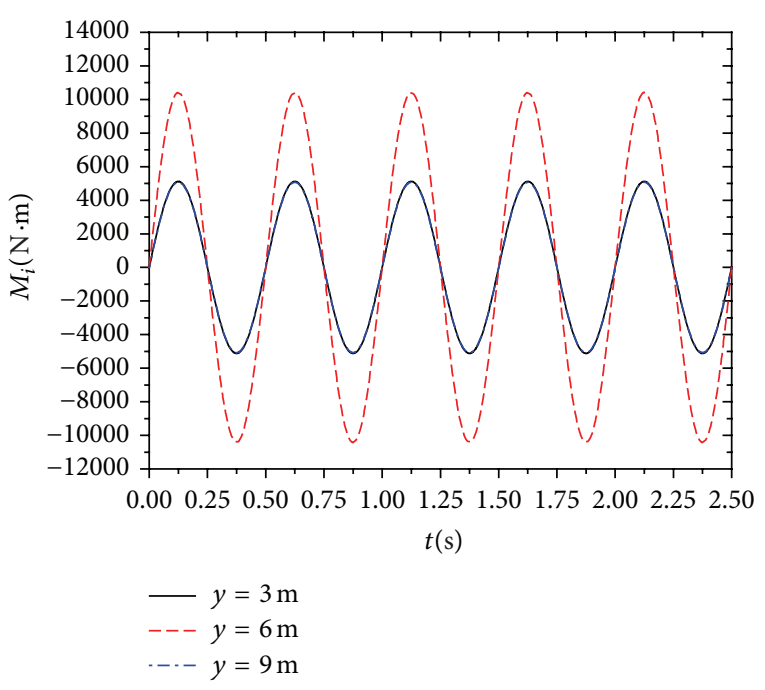

FIGURE 7: Bending moment response at midspan section of vertical beam.

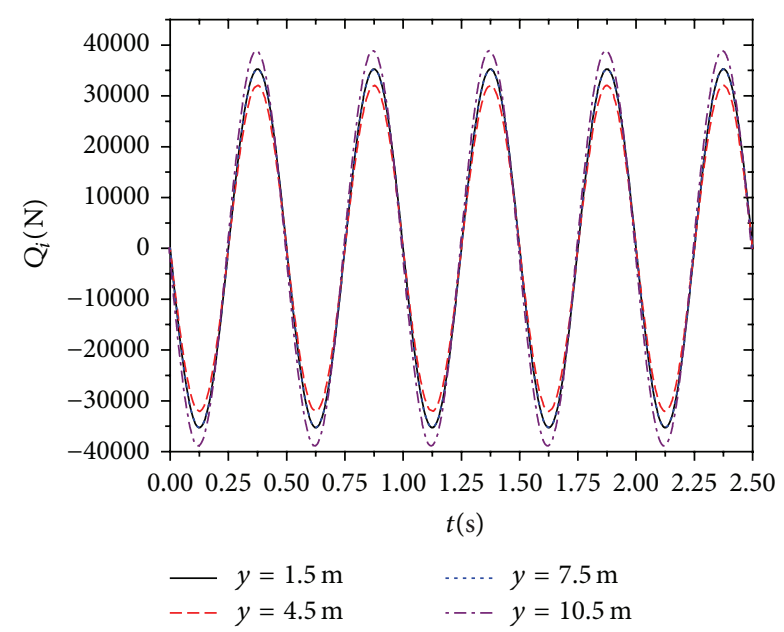

FIGURE 8: Shear force response at left hand of intersection of vertical beam.

$$
\begin{aligned}
& \left(2 w_{i}\left(j, N_{i}\right)-5 w_{i}\left(j, N_{i}-1\right)\right. \\
& \left.+4 w_{i}\left(j, N_{i}-2\right)-w_{i}\left(j, N_{i}-3\right)\right)\left(\Delta l_{i}^{2}\right)^{-1} \\
& =\left(2 w_{i+1}\left(j, N_{i}\right)-5 w_{i+1}\left(j, N_{i}+1\right)\right. \\
& \left.\quad+4 w_{i+1}\left(j, N_{i}+2\right)-w_{i+1}\left(j, N_{i}+3\right)\right)\left(\Delta l_{i+1}^{2}\right)^{-1} .
\end{aligned}
$$

The displacement response of $i$ th column grid nodes should also satisfy the following initial conditions:

$$
\begin{gathered}
w_{i}[0, k]=0 \\
\left.\frac{\partial w_{i}}{\partial t}\right|_{t=0}=\frac{w_{i}[1, k]-w_{i}[0, k]}{\Delta t}=-2 v_{0} .
\end{gathered}
$$

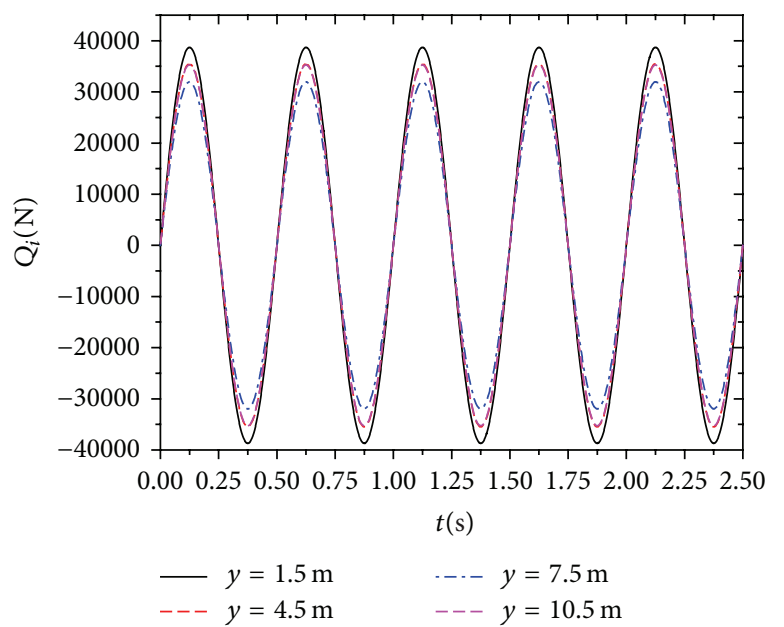

FIGURE 9: Shear force response at right hand of intersection of vertical beam.

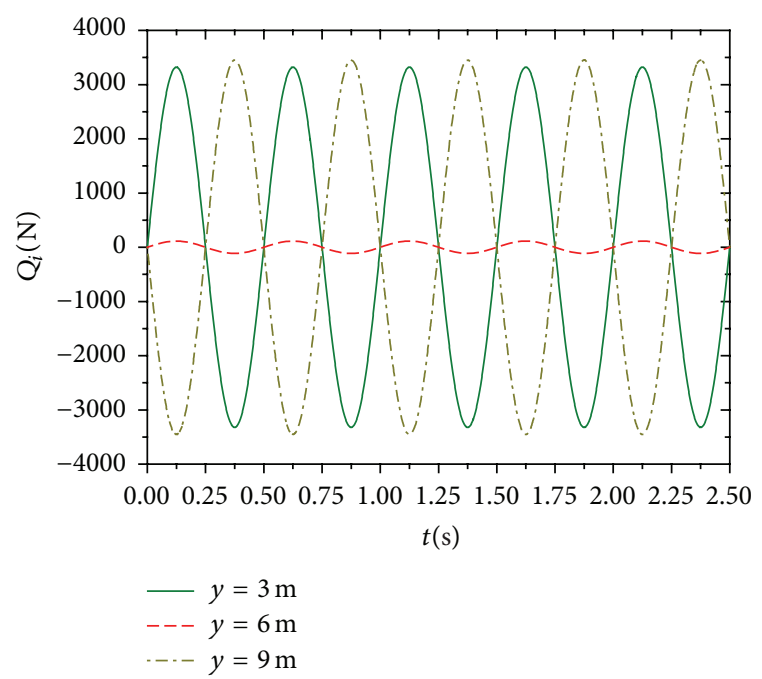

FIGURE 10: Shear force response at midspan section of vertical beam.

In (17), $v_{0}$ is the initial velocity of vertical beam. If the harmonic vibration is needed, then the initial velocity can be defined as $v_{0}=-a_{g} / \omega_{g}$.

Equations (15)-(17) can be simplified and the finite difference format for the dynamic response of vertical beam is shown as

$$
\begin{gathered}
w_{i}[0, k]=0, \\
w_{i}[1, k]=-2 \Delta t v_{0}, \\
w_{i}(j+1, k) \\
=\left(2-\frac{6 \Delta t^{2}}{\alpha_{1} \Delta l_{i}^{4}}-\frac{\alpha_{2} \Delta t^{2}}{\alpha_{1}}\right) w_{i}(j, k) \\
+\frac{\Delta t^{2}}{\alpha_{1} \Delta l_{i}^{4}}\left[4 w_{i}(j, k+1)+4 w_{i}(j, k-1)\right.
\end{gathered}
$$$$
w_{i}(j+1, k)
$$$$
=\left(2-\frac{6 \Delta t^{2}}{\alpha_{1} \Delta l_{i}^{4}}-\frac{\alpha_{2} \Delta t^{2}}{\alpha_{1}}\right) w_{i}(j, k)
$$ 


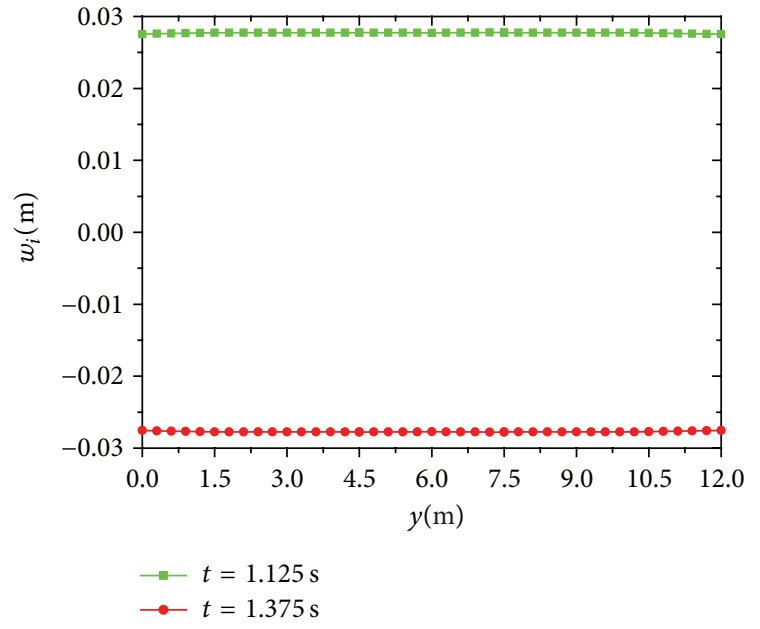

FIGURE 11: Displacement response along vertical beam at typical moment.

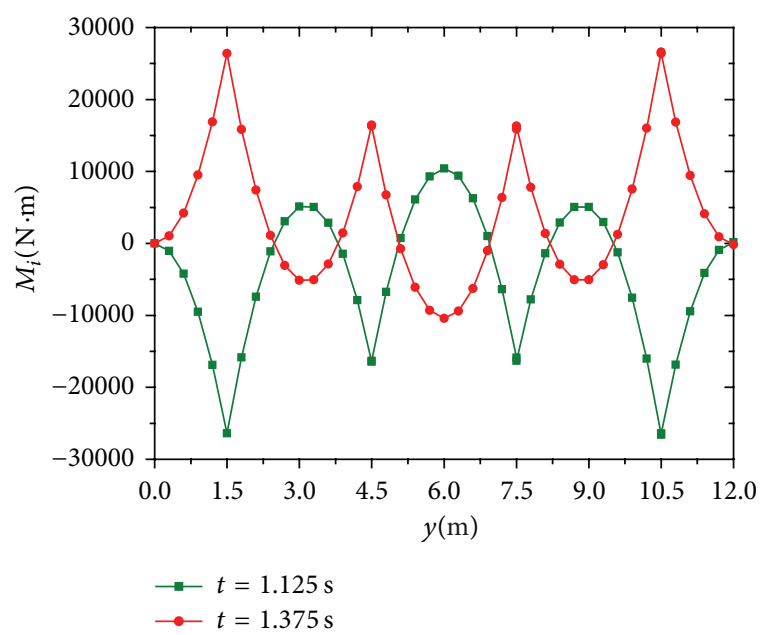

FIGURE 12: Bending moment response along vertical beam at typical moment.

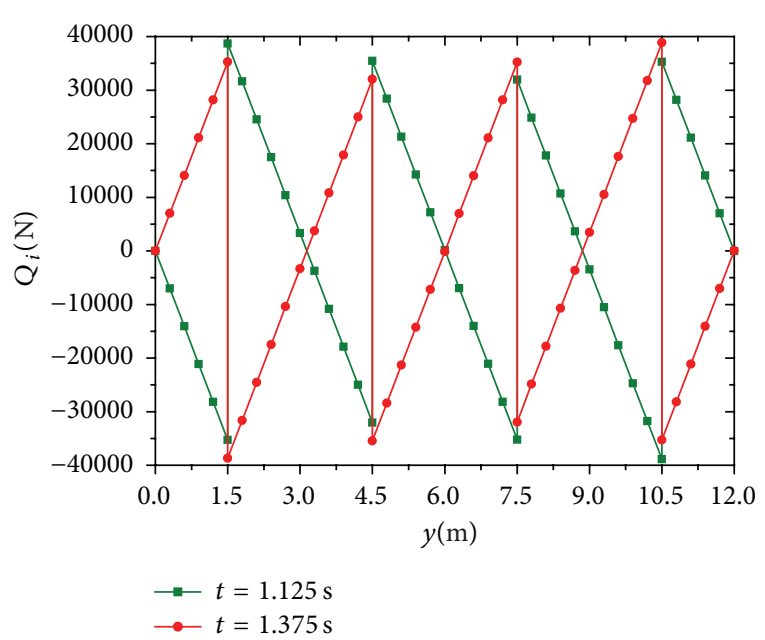

FIGURE 13: Shear force response along vertical beam at typical moment.

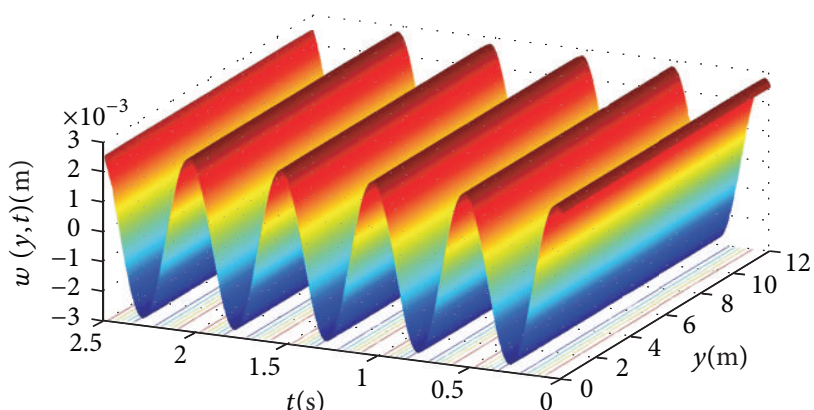

FIGURE 14: Displace response of vertical beam.

$$
\begin{array}{r}
\left.-w_{i}(j, k+2)-w_{i}(j, k-2)\right] \\
-w_{i}(j-1, k)+\Delta t^{2} \psi_{i}(k) a_{g}^{\prime}(j \Delta t)
\end{array}
$$

$$
w_{1}(j, 0)=3 w_{1}(j, 2)-2 w_{1}(j, 3)
$$$$
w_{1}(j, 1)=2 w_{1}(j, 2)-w_{1}(j, 3),
$$$$
w_{n}\left(j, N_{n}-1\right)=2 w_{n}\left(j, N_{n}-2\right)-w_{n}\left(j, N_{n}-3\right),
$$$$
w_{n}\left(j, N_{n}\right)=3 w_{n}\left(j, N_{n}-2\right)-2 w_{n}\left(j, N_{n}-3\right),
$$

$$
w_{i}\left(j, N_{i}\right)=w_{i+1}\left(j, N_{i}\right)=u_{i 2}\left(L_{i 2}, j \Delta t\right) \cos \varphi_{i},
$$$$
w_{i+1}\left(j, N_{i}+1\right)
$$

$$
=\left(\left[\frac{6 \Delta l_{i}^{2}}{\Delta l_{i+1}^{2}}+\frac{10\left(\Delta l_{i}+\Delta l_{i+1}\right)}{\Delta l_{i+1}}-6\right] w_{i}\left(j, N_{i}\right)\right.
$$$$
-7 w_{i}\left(j, N_{i}-2\right)+3 w_{i}\left(j, N_{i}-3\right)
$$$$
+\left[\frac{12 \Delta l_{i}^{2}}{\Delta l_{i+1}^{2}}+\frac{5 \Delta l_{i}}{\Delta l_{i+1}}\right] w_{i+1}\left(j, N_{i}+2\right)
$$$$
\left.-\frac{3 \Delta l_{i}^{2}}{\Delta l_{i+1}^{2}} w_{i+1}\left(j, N_{i}+3\right)\right)
$$

$$
\times\left(15\left[\frac{\Delta l_{i}}{\Delta l_{i+1}}+\frac{\Delta l_{i}^{2}}{\Delta l_{i+1}^{2}}\right]\right)^{-1}
$$

$$
\begin{aligned}
w_{i}\left(j, N_{i}-1\right) & \frac{2\left(\Delta l_{i}+\Delta l_{i+1}\right)}{3 \Delta l_{i+1}} w_{i}\left(j, N_{i}\right)+\frac{1}{3} w_{i}\left(j, N_{i}-2\right) \\
& -\frac{\Delta l_{i}}{\Delta l_{i+1}} w_{i+1}\left(j, N_{i}+1\right)+\frac{\Delta l_{i}}{3 \Delta l_{i+1}} w_{i+1}\left(j, N_{i}+2\right) .
\end{aligned}
$$

According to the finite difference method mentioned above, for a specific engineering problem, the displacement of grid points in the initial and the first row can be calculated by (18). For the second row, the displacement of all the grid nodes in interior columns can be decided by (19), the grid nodes in exterior boundary columns and their adjacent columns (columns $0,1, N_{n}-1$, and $N_{n}$ ) can be decided by (20) and based on (21), the node displacement of all the interior boundary adjacent columns can be achieved. Likewise, the 


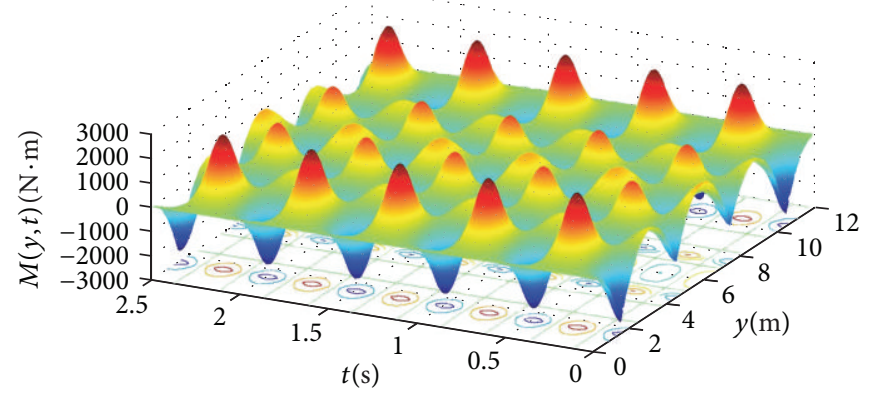

FIGURE 15: Bending moment response of vertical beam.

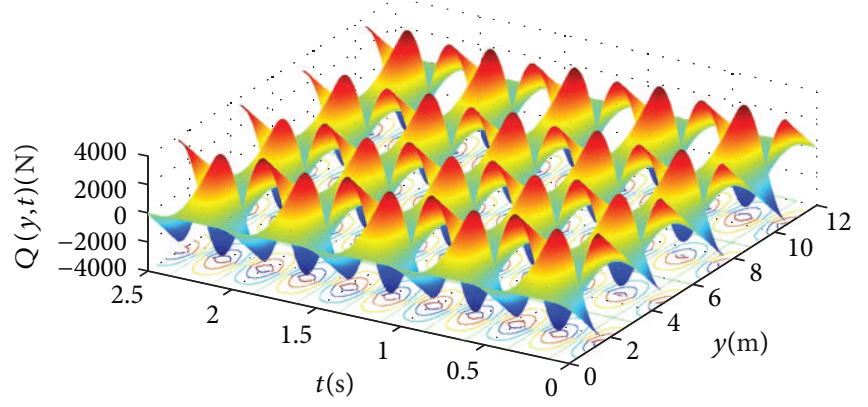

FIGURE 16: Shear force response of vertical beam.

displacement for all the grid nodes from row 3 to row $M$ can be decided.

After getting the displacement of all grid nodes, according to $M_{i}=E I\left(\partial^{2} w_{i} / \partial y^{2}\right)$ and $Q_{i}=E I\left(\partial^{3} w_{i} / \partial y^{3}\right)$, the numerical solutions to the bending moment and shear force response of vertical beam can be obtained by conducting the finite difference to the displacement of all grid nodes again.

4.2. Comparative Analysis and Discussion. Similarly, based on previous case study, the slope is considered under horizontal harmonic seismic loading and with anchoring system damping neglected or considered, respectively. The numerical solutions to the dynamic response of vertical beam can be achieved by using finite difference method and MATLAB programming, and $r_{i}=\Delta t / \Delta^{2} l_{i}=0.00001 /(0.25 \times 0.25)=$ $0.00016 \leq \sqrt{\alpha_{1}}=0.002(i=1 \sim 5)$.

In order to verify the correctness of the two methods above, the solutions of intersection $y=1.5 \mathrm{~m}$ by different methods and with damping effect neglected or considered are shown in Figures 18 and 19.

When damping effect is neglected, according to Figure 18, it is found that the calculation results by the three methods share similar vibration trend and only show slight difference in amplitude; generally speaking, the finite element solution mentioned previously is the smallest due to the size of finite difference grids and it achieved by method of Literature [7] is second because of the calculated amount of high order vibration mode, and the theoretical solution mentioned previously is the largest and the most reasonable, but the accuracy of two previous methods can be, respectively, improved by decreasing grid ratio and increasing the calculated amount so that the results of the three methods show more excellent agreement, which indicates the reasonability and feasibility of two proposed methods under the condition of neglecting damping effect.

When damping effect is considered, as shown in Figure 19, the proposed theoretical solution and literature [7] solution are also well consistent and the former is slightly larger than the latter. Through the comparison and analysis of Figures 14, 15, 16, and 19, it is found that when using the finite difference method, the attenuation characteristic of vertical beam response can be clearly shown in the first second and then with the passing of time the vibration mode is gradually degenerated into constant state, while no attenuation characteristic is shown and the calculating result keeps steady state all the time if using the theoretical methods. So in the first second, the calculating results used the finite difference method and theoretical methods have considerable difference, which is attributed to the theoretical solution based on steady-state complex method. Specifically, the calculating result using finite difference method is larger than the result using theoretical methods in the first second. After the first second, the vibration pattern and the amplitude are consistent by using these three methods. Besides, the finite difference method not only can demonstrate the attenuation characteristic of amplitude in terms of magnitude in the damping anchorage system, but also can illustrate the attenuation characteristic of amplitude in terms of time, and its calculating result will be more reliable and reasonable.

Generally speaking, the finite difference method can not only be applied to the dynamic response analysis of frame supporting structure under harmonic earthquake, but 


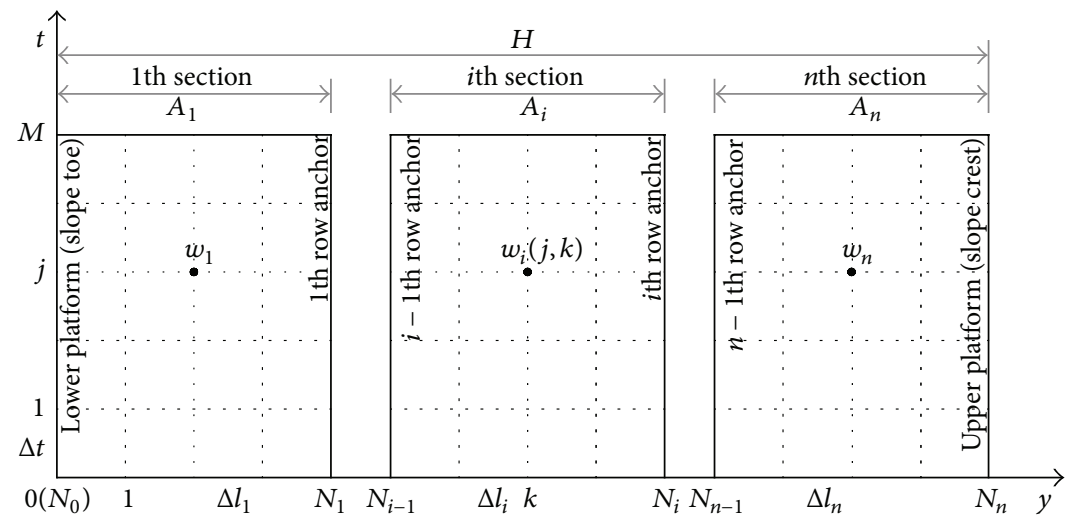

FIGURE 17: Finite difference grids of vertical beam.

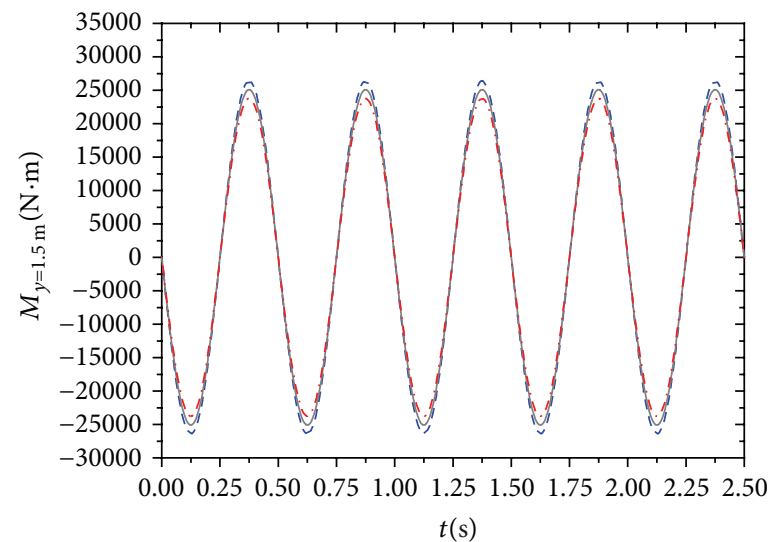

- - Proposed theoretical method

-.- Proposed finite difference method

— Literature [7] method

(a) Bending moment response at intersection $y=1.5 \mathrm{~m}$

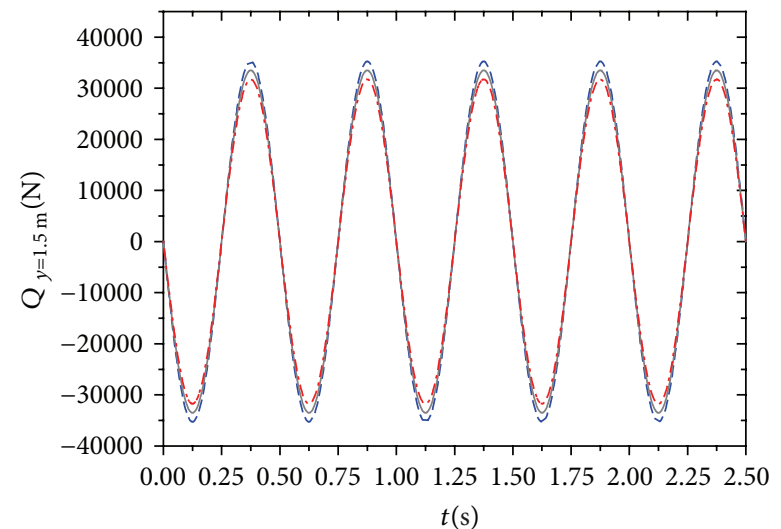

- - - Proposed theoretical method

... Proposed finite difference method

— Literature [7] method

(b) Shear force response at left hand of intersection $y=1.5 \mathrm{~m}$

FIGURE 18: Solutions of vertical beam with damping effect neglected by different methods.

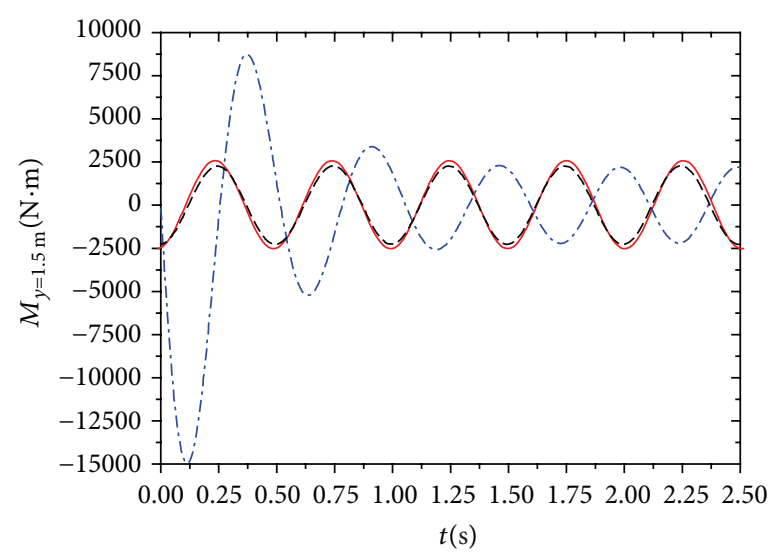

$t(\mathrm{~s})$

— Proposed theoretical method

-..-. Proposed finite difference method

--- Literature [7] method

(a) Bending moment response at intersection $y=1.5 \mathrm{~m}$

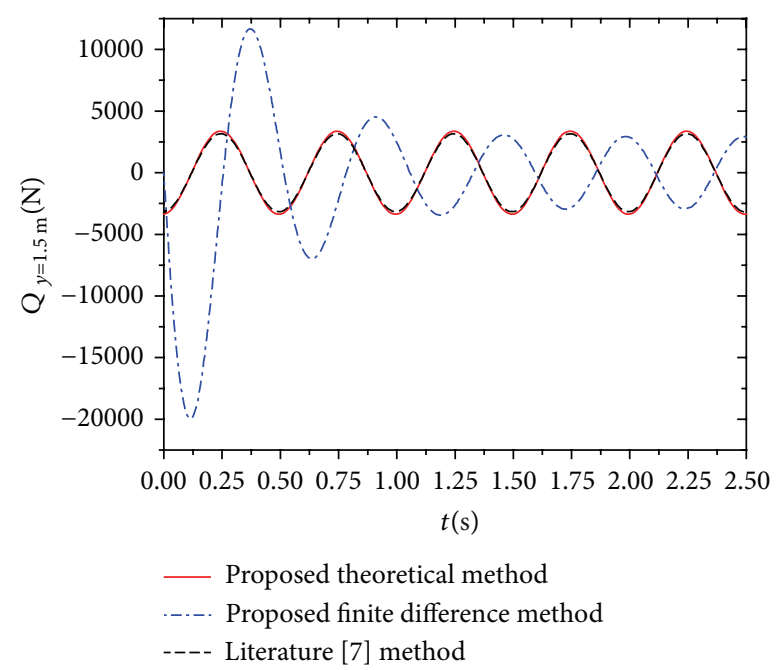

(b) Shear force response at left hand of intersection $y=1.5 \mathrm{~m}$

FIGURE 19: Solutions of vertical beam with damping effect considered by different methods. 
also be used to the analysis of random seismic loading. Besides, it can demonstrate the attenuation characteristics of amplitude in terms of both magnitude and time; in other words, it can indicate the damping effect better. Therefore, the calculating result obtained by finite difference method would be more approximate to actual conditions and hence be more reliable and reasonable. However, the analytical method is only confined to the analysis of frame supporting structure under harmonic earthquake. Besides, it can only reflect the attenuation characteristics of amplitude in terms of magnitude and the peak value of mechanical response got by analytical method would be underestimated; as a result potential hazards may be brought about. What is more, based on steady-state complex method, its mechanical responses have initial phase values, which are clearly contradictory to the reality.

\section{Conclusions}

Through the dynamic response analysis of frame supporting structure of slope under harmonic forced vibration, the conclusion follows.

(1) Based on certain assumptions and force analysis of segment of infinitesimal length, the dynamic governing equation of vertical beam under forced vibration is derived and the analytical solutions to the dynamic response of vertical beam under harmonic forced vibration are obtained. Meanwhile, according to finite difference method, the finite difference format and corresponding calculating procedure for dynamic response of vertical beam under forced vibration are established and finally programmed by using MATLAB language.

(2) In the case studies, three methods have been used in the dynamic response analysis and with damping effect neglected or considered, respectively. The feasibility and correctness of the two proposed methods have been examined, and the characteristic of each method has been summarized.

(3) Under horizontal harmonic seismic loading, the mechanical response of vertical beam would synchronize correspondingly. Its response law shows agreement with the one of two-end cantilevered continuous beam, and the anchor to the frame supporting structure, is just like the bearing to the continuous beam. In specific engineering design, the factors such as mechanical characteristic of vertical beam and slope supporting effect of anchors should also be considered.

(4) Compared with analytical solutions, the finite difference method can simulate the dynamic response of frame supporting structure under various earthquake conditions, and it also can indicate the damping effect of anchorage system very well; hence the calculating result obtained is more reliable and reasonable. Moreover, the finite difference method is achieved based on programming calculation, its calculation speed and accuracy can be guaranteed, and hence it has broad application prospect.

\section{Conflict of Interests}

The authors declares that there is no conflict of interests regarding the publication of this paper.

\section{Acknowledgments}

This paper is supported by Project (51308273, 41372307) supported by National Natural Science Foundation of China and Project (2010(A)06-b) supported by Science and Technology Project supported by Yunan Provincial Communication Department.

\section{References}

[1] L. K. Cheng, "Present status and development of ground anchorages," China Civil Engineering Journal, vol. 34, no. 3, pp. 7-16, 2001 (Chinese).

[2] D. P. Zhou, J. J. Zhang, and Y. Tang, "Seismic damage analysis of road slopes in Wenchuan earthquake," Chinese Journal of Rock Mechanics and Engineering, vol. 29, no. 3, pp. 565-576, 2010 (Chinese).

[3] M. Yang, H. T. Hu, C. J. Lu et al., "Calculation of internal forces for prestressed anchor cable frame used in reinforced roadcut soil slope," Chinese Journal of Rock Mechanics and Engineering, vol. 21, no. 9, pp. 1383-1386, 2002 (Chinese).

[4] Y. H. Tian, J. K. Liu, and Y. F. Zhang, "Internal force calculation of prestressed anchor cable frame by finite difference method," Journal of Beijing Jiaotong University, vol. 31, no. 4, pp. 22-25, 2007 (Chinese).

[5] G. H. Lin, "Model test of anchor grid beam for cut slope," Technology of Highway and Transport, vol. 3, pp. 19-22, 2004 (Chinese).

[6] B. L. Zhu, M. Yang, H. T. Hu et al., “Testing study on internal forces for prestressed anchor cable frame in reinforced soil slope," Chinese Journal of Rock Mechanics and Engineering, vol. 24, no. 4, pp. 697-702, 2005 (Chinese).

[7] J. H. Dong, Y. P. Zhu, Y. Zhou et al., "Dynamic calculation model and seismic response for frame supporting structure with prestressed anchors," Science China Technological Sciences, vol. 53, no. 7, pp. 1957-1966, 2010.

[8] S. Çatal, "Solution of free vibration equations of beam on elastic soil by using differential transform method," Applied Mathematical Modelling, vol. 32, no. 9, pp. 1744-1757, 2008.

[9] M. Balkaya, M. O. Kaya, and A. Sağlamer, "Analysis of the vibration of an elastic beam supported on elastic soil using the differential transform method," Archive of Applied Mechanics, vol. 79, no. 2, pp. 135-146, 2009.

[10] M. Ansari, E. Esmailzadeh, and D. Younesian, "Frequency analysis of finite beams on nonlinear KelvinVoight foundation under moving loads," Journal of Sound and Vibration, vol. 330, no. 7, pp. 1455-1471, 2011.

[11] O. Demirdag and Y. Yesilce, "Solution of free vibration equation of elastically supported Timoshenko columns with a tip mass by differential transform method," Advances in Engineering Software, vol. 42, no. 10, pp. 860-867, 2011. 
[12] M. H. Yas and N. Samadi, "Free vibrations and buckling analysis of carbon nanotube-reinforced composite Timoshenko beams on elastic foundation," International Journal of Pressure Vessels and Piping, vol. 98, pp. 119-128, 2012.

[13] R. A. Jafari-Talookolaei, M. Abedi, M. H. Kargarnovin et al., "An analytical approach for the free vibration analysis of generally laminated composite beams with shear effect and rotary inertia," International Journal of Mechanical Sciences, vol. 65, no. 1, pp. 97-104, 2012.

[14] S. W. Qi, F. Q. Wu, F. Z. Yan et al., Dynamic Response Analyses of Rock Slope, Science Press, Beijing, China, 2007, Chinese.

[15] J. H. Dong and Y. P. Zhu, "Dynamic calculation model and seismic response for the system of soil nailing and surrounding soil," Chinese Journal of Theoretical and Applied Mechanics, vol. 41, no. 2, pp. 236-242, 2009.

[16] Z. L. Xu, Elasticity, Higher Education Press, Beijing, China, 2009, Chinese. 


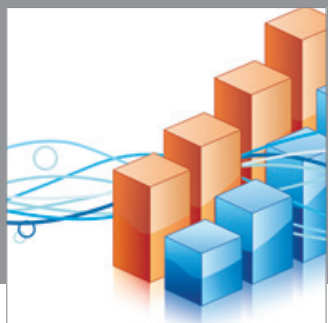

Advances in

Operations Research

mansans

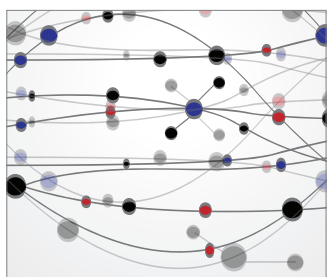

The Scientific World Journal
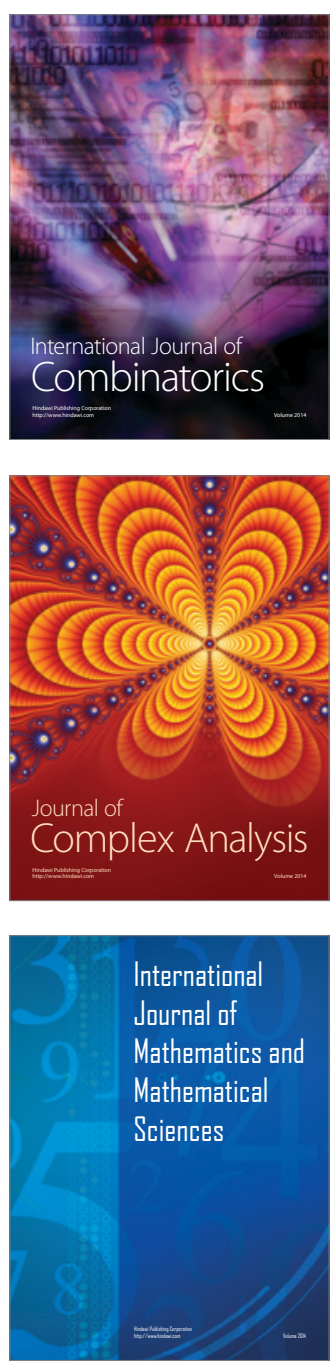
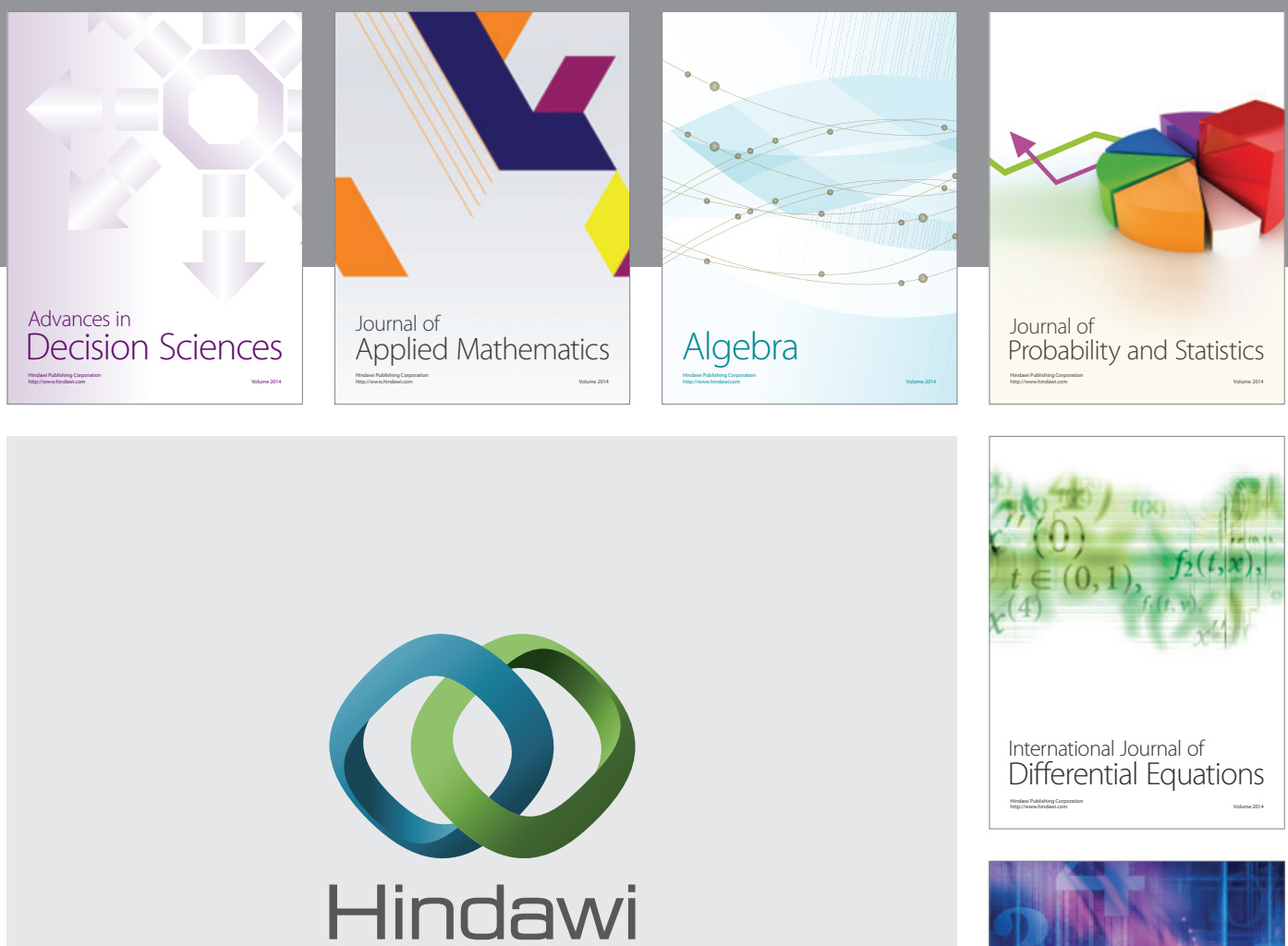

Submit your manuscripts at http://www.hindawi.com
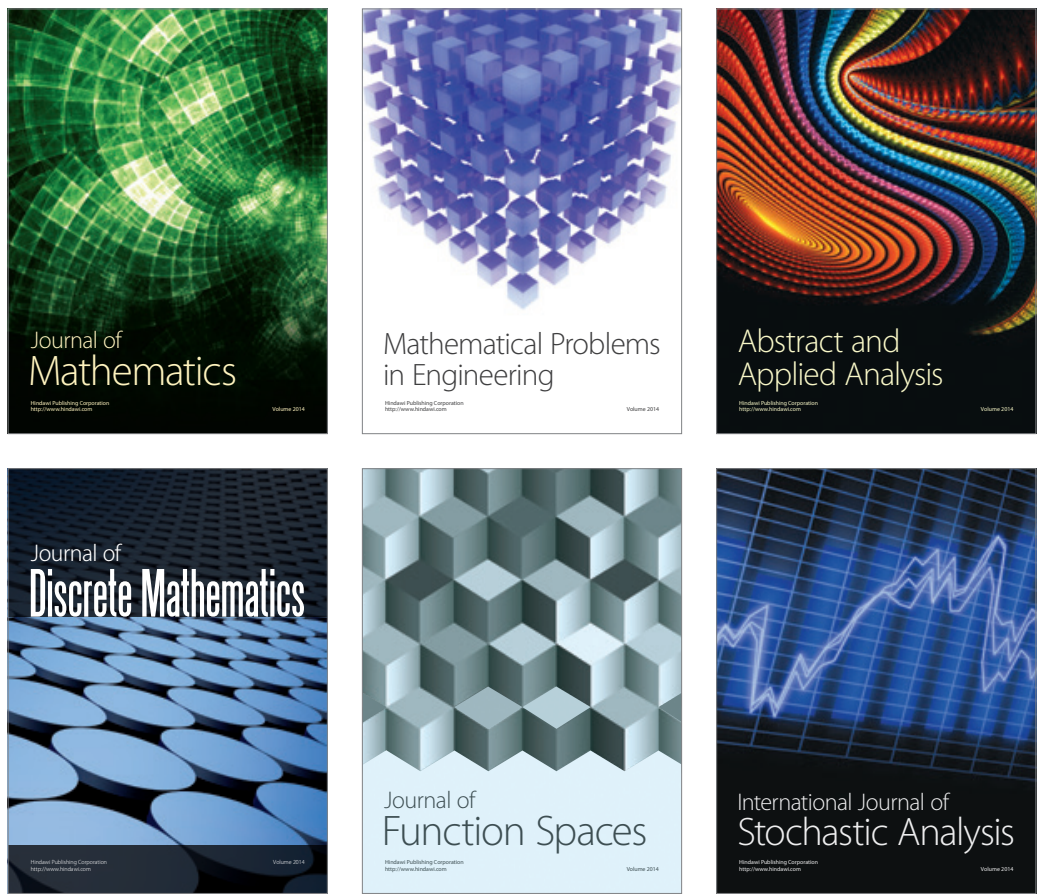

Journal of

Function Spaces

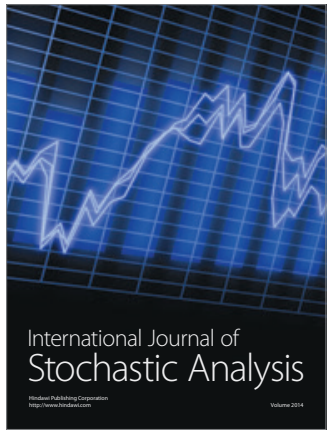

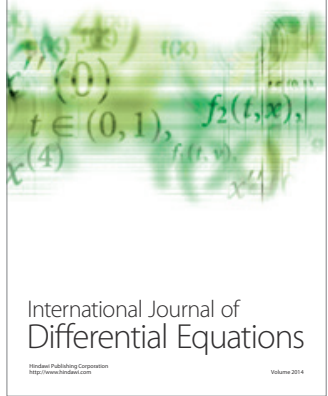
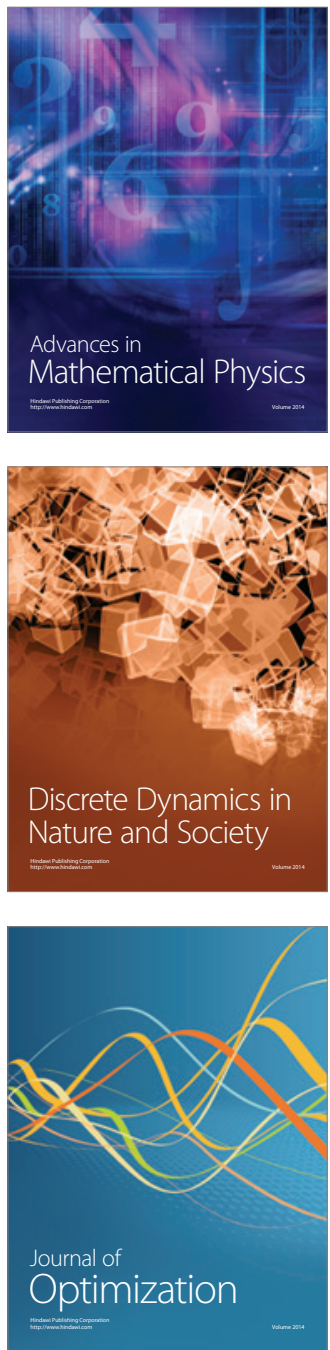